\title{
A Practical Statistical Polychromatic Image Reconstruction for Computed Tomography Using Spectrum Binning
}

\author{
Meng $\mathrm{Wu}^{a, b}$, Qiao Yang $^{c}$, Andreas Maier ${ }^{c}$, and Rebecca Fahrig ${ }^{b}$ \\ ${ }^{a}$ Electrical Engineering Department, Stanford University, Stanford, USA; \\ ${ }^{b}$ Radiology Department, Stanford University, Stanford, USA; \\ ${ }^{c}$ Pattern Recognition Lab, FAU Erlangen-Nuremberg, Germany
}

\begin{abstract}
Polychromatic statistical reconstruction algorithms have very high computational demands due to the difficulty of the optimization problems and the large number of spectrum bins. We want to develop a more practical algorithm that has a simpler optimization problem, a faster numerical solver, and requires only a small amount of prior knowledge. In this paper, a modified optimization problem for polychromatic statistical reconstruction algorithms is proposed. The modified optimization problem utilizes the idea of determining scanned materials based on a first pass FBP reconstruction to fix the ratios between photoelectric and Compton scattering components of all image pixels. The reconstruction of a density image is easy to solve by a separable quadratic surrogate algorithm that is also applicable to the multi-material case. In addition, a spectrum binning method is introduced so that the full spectrum information is not required. The energy bins sizes and attenuations are optimized based on the true spectrum and object. With these approximations, the expected line integral values using only a few energy bins are very closed to the true polychromatic values. Thus both the problem size and computational demand caused by the large number of energy bins that are typically used to model a full spectrum are reduced. Simulation showed that three energy bins using the generalized spectrum binning method could provide an accurate approximation of the polychromatic X-ray signals. The average absolute error of the logarithmic detector signal is less than 0.003 for a $120 \mathrm{kVp}$ spectrum. The proposed modified optimization problem and spectrum binning approach can effectively suppress beam hardening artifacts while providing low noise images.
\end{abstract}

Keywords: Iterative reconstruction, Beam hardening artifact, CT, Spectrum binning, Polychromatic X-ray

\section{INTRODUCTION}

Beam hardening artifacts in computed tomography $(\mathrm{CT})$ images are caused by the energy dependent attenuation coefficients of materials as well as the polychromatic X-ray source. When a polychromatic X-ray beam passes through a larger thickness of material, the low energy X-rays will be attenuated more than the high energy $\mathrm{X}$-rays. Thus, the effective amounts of attenuation is no longer linear with the material thickness, and the Beer's Law does not hold anymore. In addition, the energy dependent attenuation coefficients differ between materials. For example, bone will block more low energy X-rays than water. The water correction is not sufficient to remove the shadows between the bones in the FBP reconstructed images. ${ }^{1}$

Many beam hardening correction (BHC) algorithms have been developed for X-ray CT, both analytical ${ }^{1}$ and iterative. ${ }^{2-6}$ The water correction method uses a polynomial fitting (or lookup table ) to convert detector signals from polychromatic to monochromatic. The water correction method is based on the assumption that the human body is mainly water, thus the algorithm is not efficient when the object contains other dense materials such as bone. Joseph and Spital ${ }^{1}$ proposed a correction algorithm that uses the precomputed image to identify the bone and soft tissue and then modify the projections with two-material dependent lookup tables. However, this algorithm relies on the precomputed FBP image that has beam hardening artifacts. FBP image may not be able to provide an accurate estimation of the materials and density and so the one step beam hardening correction is usually not perfect. Yan et. al. proposed a quasi-iterative algorithm also using the idea that the scanned object can be expressed as a mixture of two known substances. ${ }^{7}$ Experimental results showed that the algorithm is much more robust and accurate than one step correction.

De-Man et. al. proposed a maximum likelihood based reconstruction algorithm ${ }^{3}$ (IMPACT), in which the energy dependent attenuation coefficients of materials were decomposed into linear combinations of photoelectric 
and Compton scattering components. The weights of the two effects in each voxel are adjusted based on the effective linear attenuation coefficient within each iteration. Another approach was proposed by Elbakri et. al., which reconstructs a density map from pre-segmented base substance images. ${ }^{2,8}$ Both of the statistical algorithms can effectively correct beam hardening artifacts while benefiting from the noise reduction from the maximum likelihood estimator. The main drawback of IMPACT is the complicated optimization and the requirement all the photoelectric and Compton scattering relationships of all substances that are known prior to the scan. The segmentation-based algorithms have more sophisticated optimization, but are limited by the number of substances when the lookup table of the gradient is used. Moreover, both of the approaches directly use a known single full X-ray spectrum and have high computational cost or memory usage. In this study, we aim to simplify the algorithms with a combined objective function and use a minimal amount of spectrum information.

\section{METHODS}

\subsection{Modified Optimization Problem}

The expected detector signal of a polychromatic X-ray beam is a combination of multiple small energy bins that follows Beer's law:

$$
\bar{Y}_{i}=\int_{\varepsilon} b_{i}(\varepsilon) \exp \left(-\int_{L_{i}} \mu(\varepsilon) \mathrm{d} l\right) \mathrm{d} \varepsilon
$$

where $b_{i}(\varepsilon)$ denotes the unattenuated signal of detector pixel $i$ at energy $\varepsilon$, and $\mu(\varepsilon)$ denotes the energy dependent linear attenuation coefficient of the object. The measured detector signal follows the Poisson (or Compound Poisson depending on the detector) statistical model:

$$
Y_{i} \sim \operatorname{Poisson}\left\{\bar{Y}_{i}\right\}+n_{i}
$$

where $n_{i}$ denotes the additional signal, including scattering and electronic noise, that is usually modeled by a Gaussian random variable $\mathbf{N}\left(r_{i}, \sigma_{i}\right)$. The linear integral of attenuation coefficients (sinogram) under monochromatic assumption can be computed by

$$
\hat{p}_{i}=\log \left(\frac{b_{i}}{Y_{i}-r_{i}}\right) .
$$

Based on the assumption of the Poisson measurement model and ignoring the electronic noise, we can reconstruct the image through minimization of the log-likelihood objective function ${ }^{2,3}$ as

$$
\Psi=-\sum_{i=1}^{I}\left(Y_{i} \ln \left(\bar{Y}_{i}\right)-\bar{Y}_{i}\right)+\beta \mathbf{R}(\mu),
$$

where the argument $\mathbf{R}$ is the penalty function of the image roughness.

For any particular substance, the energy dependent mass attenuation coefficient can by approximated by a linear combination of photoelectric and Compton scattering components ${ }^{3}$ (at low X-ray energies), such as

$$
m_{k}(\varepsilon)=\phi_{k} \Phi(\varepsilon)+\theta_{k} \Theta(\varepsilon)
$$

where $\Phi(\varepsilon)$ and $\Theta(\varepsilon)$ denote base functions of the photoelectric and Compton scattering components, and $\phi_{k}$ and $\theta_{k}$ are the amounts of each of the two components for substance $k$. Then the attenuation coefficients $\mu_{j}(\varepsilon)$ in (1) can be represented as:

$$
\begin{aligned}
\mu_{j}(\varepsilon) & =\sum_{k=1}^{K}\left(\phi_{k} f_{j}^{k} \Phi(\varepsilon)+\theta_{k} f_{j}^{k} \Theta(\varepsilon)\right) \rho_{j} \\
& =\left(\Phi(\varepsilon) \sum_{k=1}^{K} \phi_{k} f_{j}^{k}+\Theta(\varepsilon) \sum_{k=1}^{K} \theta_{k} f_{j}^{k}\right) \rho_{j} \\
& =\left(\phi_{j} \Phi(\varepsilon)+\theta_{j} \Theta(\varepsilon)\right) \rho_{j},
\end{aligned}
$$


where $f_{j}^{k}$ is the fraction of the $k$ th material in the $j$ th voxel, $\phi_{j}$ and $\theta_{j}$ is the total amount of each of the two components in the $j$ th voxel, and $\rho_{j}$ is the density of image pixel $j$. Therefore, the segmentation-based approach is equivalent to fixing the ratio of the photoelectric and Compton scattering components. Then the number of material substances can be arbitrarily large without causing an increase in the number of projections and complexity in computing the gradients.

One difference between the segmentation based method and IMPACT is whether or not the ratio is adjusted in the iteration based on the previous iteration results. Given the fact that segmentation-based methods have very good image quality, and are easier to design with respect to the optimization solver, ${ }^{2,5,8}$ we propose to use a modified optimization problem, in which $\bar{Y}_{i}$ in (4) is obtained by

$$
\begin{aligned}
\bar{Y}_{i} & =\int_{\varepsilon} b_{i}(\varepsilon) \exp \left(-\int_{L_{i}}\left(\phi_{j} \Phi(\varepsilon)+\theta_{j} \Theta(\varepsilon)\right) \rho_{j} \mathrm{~d} l\right) \mathrm{d} \varepsilon \\
& =\int_{\varepsilon} b_{i}(\varepsilon) \exp \left(-\Phi(\varepsilon) \int_{L_{i}} \phi_{j} \rho_{j} \mathrm{~d} l-\Theta(\varepsilon) \int_{L_{i}} \theta_{j} \rho_{j} \mathrm{~d} l\right) \mathrm{d} \varepsilon .
\end{aligned}
$$

The ratio of the coefficients of the photoelectric and Compton scattering components are precomputed and fixed using an FBP reconstruction and prior knowledge. The idea is similar to the approach in. ${ }^{4}$ Therefore, the algorithm can be applied to datasets with multiple materials without significantly increasing computational costs (forward and backward projections), while having a relatively easier optimization problem since only the density map is changed in the iterations.

\subsection{Spectrum Binning}

It has been shown that the spectrum information need not to be known in full detail for good BHC results. 3 - 20 energy bins have been used both in simulations and phantom studies. ${ }^{2-4}$ In this study, we want to find a strategy that uses the least amount of information (energy bins) to effectively represent the full spectrum.

The threshold-based spectrum binning (SB) method simplifies the detector-dependent spectrum with a few wider energy bins by thresholding. The unattenuated scan factor of energy bin $s$ is computed by integrating the spectrum between the lower and upper bounds.

$$
B_{s}=\int_{\tau_{s}}^{\tau_{s+1}} b(\varepsilon) \mathrm{d} \varepsilon
$$

where $\tau_{s}$ is the cutoff threshold between bins $s-1$ and $s$. The sum of the spectrum bins is equal to the integral of the original spectrum

$$
\sum_{s=1}^{S} B_{s}=\int_{\varepsilon_{\min }}^{\varepsilon_{\max }} b(\varepsilon) \mathrm{d} \varepsilon .
$$

For a given amount of two components $\left(\phi_{t}, \theta_{t}\right)$, the true and approximated expected detector signals in photon counts are

$$
\begin{aligned}
\bar{Y}^{\text {full }}\left(\phi_{t}, \theta_{t}\right) & =\int_{\varepsilon} b(\varepsilon) \exp \left(-\Phi(\varepsilon) \phi_{t}-\Theta(\varepsilon) \theta_{t}\right) \mathrm{d} \varepsilon \\
\bar{Y}^{S}\left(\phi_{t}, \theta_{t}, \tau\right) & =\sum_{s=1}^{S} B_{s} \exp \left(-\Phi_{s} \phi_{t}-\Theta_{s} \theta_{t}\right) .
\end{aligned}
$$

$\Phi_{s}$ and $\Theta_{s}$ are the values of functions of photoelectric and Compton scattering components at the average energy of each bin. To find the "optimal" threshold, we can minimize the total distance over vector pairs of $\left(\underline{\phi}_{t}, \underline{\theta}_{t}\right)$ that are spaced over the equivalent range of materials, for example, $[0,40] \mathrm{cm}$ soft tissue and $[0,10] \mathrm{cm}$ bone.

$$
\tau^{*}=\underset{\tau}{\operatorname{argmin}}\left\|\log \left(\bar{Y}^{\text {full }}\left(\underline{\phi}_{t}, \underline{\theta}_{t}\right)\right)-\log \left(\bar{Y}^{S}\left(\underline{\phi}_{t}, \underline{\theta}_{t}, \tau\right)\right)\right\|_{p}
$$

In this study, we used the $L_{1}$ distance as the objective function for selecting the spectrum binning thresholds. Our numerical simulation has shown that use of $L_{1}$ distance produces less beam hardening artifact than $L_{2}$ or $L_{\text {inf }}$ distance. 


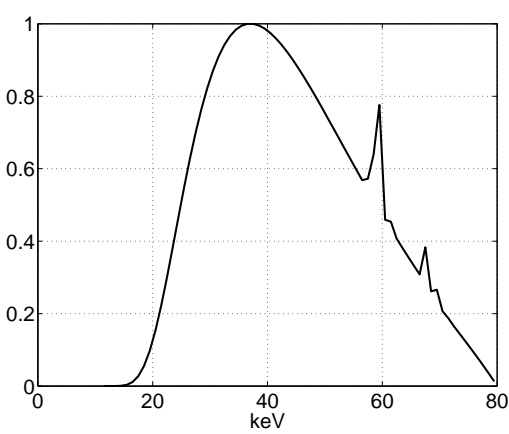

(a) $80 \mathrm{kVp}$ spectrum

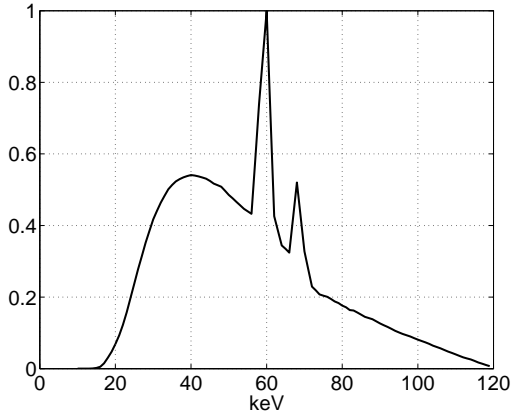

(b) $120 \mathrm{kVp}$ spectrum

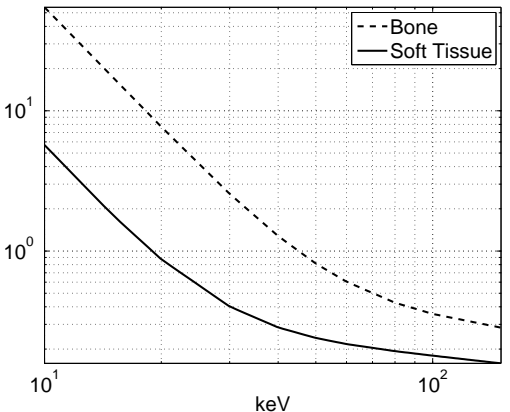

(c) soft tissue

Figure 1: Normalized $80 \mathrm{kVp}$ and $120 \mathrm{kVp}$ spectra and attenuation coefficients of soft tissue and bone.

\subsection{Generalized spectrum binning}

Only minimizing over the binning thresholds and using the effective attenuation coefficients for each energy bin may be sub-optimal. Therefore, a generalized spectrum binning (GSB) approach that has freedoms in both the bin sizes $B_{s}$ and the values of $\Phi_{s}$ and $\Theta_{s}$ is proposed. The energy bin sizes only need to satisfy the following constraints:

$$
\sum_{s=1}^{S} B_{s}=\int_{\varepsilon_{\min }}^{\varepsilon_{\max }} b(\varepsilon) \mathrm{d} \varepsilon, \quad B_{s}>0 \quad \text { for } \quad s=1,2,3, \ldots S .
$$

The optimal values of the bin sizes $B_{s}, \Phi_{s}$ and $\Theta_{s}$ values are determined by solving

$$
\underset{B_{s}, \Phi_{s}, \Theta_{s}}{\operatorname{argmin}}\left\|\log \left(\bar{Y}^{\text {full }}\left(\underline{\phi}_{t}, \underline{\theta}_{t}\right)\right)-\log \left(\bar{Y}^{S}\left(\underline{\phi}_{t}, \underline{\theta}_{t}, B_{s}, \Phi_{s}, \Theta_{s}\right)\right)\right\|_{1},
$$

where $\bar{Y}^{S}$ is computed using Eqn. (10). However, the problem described by Eqn. (13) is non-convex and it is hard to find the global minimum. We exhaustively searched over bin sizes $B_{s}$ and used the results from spectrum binning as initialization. A gradient descent method is used to find the local minimal distance. The minimization results may not be a global minimum, but the distance is much smaller than is achieved using thresholds only.

\section{SIMULATIONS}

A numerical phantom consisting of two kinds of materials is used in the simulation. The phantom is $24 \mathrm{~cm}$ in diameter soft tissue basis with four $3.2 \mathrm{~cm}$ - diameter cortical bone inserts. Material data was obtained from the NIST X-ray Attenuation Database. ${ }^{9}$ The polychromatic projection data is simulated with $80 \mathrm{kVp}$ (mean: 54.7 $\mathrm{keV}$, standard deviation $19.9 \mathrm{keV}$ ) and $120 \mathrm{kVp}$ (mean: $54.7 \mathrm{keV}$, standard deviation $19.9 \mathrm{keV}$ ) tube voltages (Figure 1). The source-to-axis distance is $100 \mathrm{~cm}$ and the axis-to-detector distance is $50 \mathrm{~cm}$. The flat-panel detector pixel has a spacing of $0.8 \mathrm{~mm}$ with a total of $2 \times 10^{6}$ photons / pixel in the unattenuated scan. 640 projections were simulated over $360^{\circ}$. The reconstructed image has $320 \times 320$ pixels with spacing of $0.8 \mathrm{~mm} \times$ $0.8 \mathrm{~mm}$.

To compare our proposed algorithm with current standard methods, the images were also reconstructed with the following algorithms:

(a) FBP water correction (FBP-WC),

(b) FBP water and bone correction (FBP-JS),

(c) Penalized weighted leased squares with water corrected data (PWLS),

(d) Segmentation-based polychromatic statistical reconstruction with full spectrum knowledge (PSR-full),

(e) Proposed physics-based polychromatic reconstruction using threshold-based spectrum binning (PSRSB2 and PSR-SB3). 


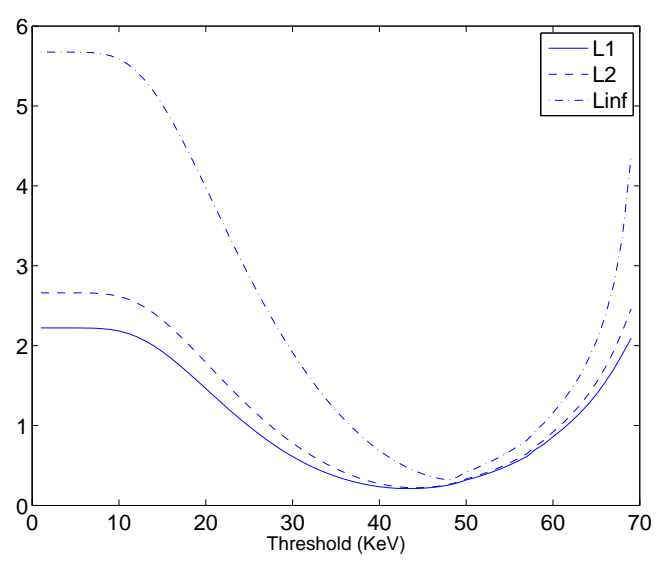

(a)

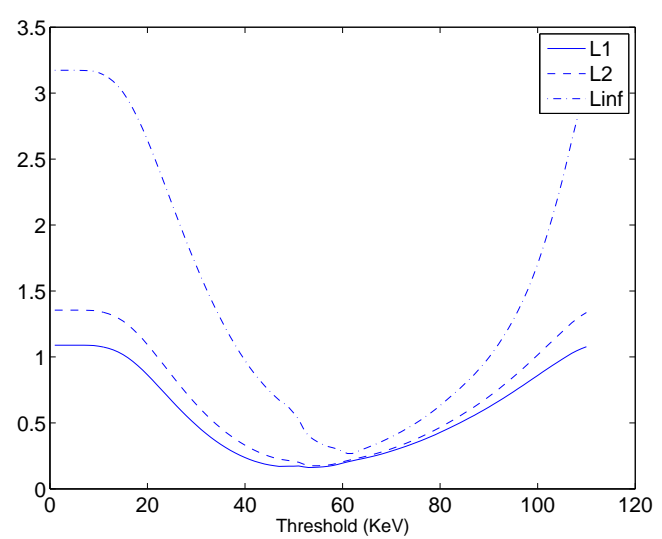

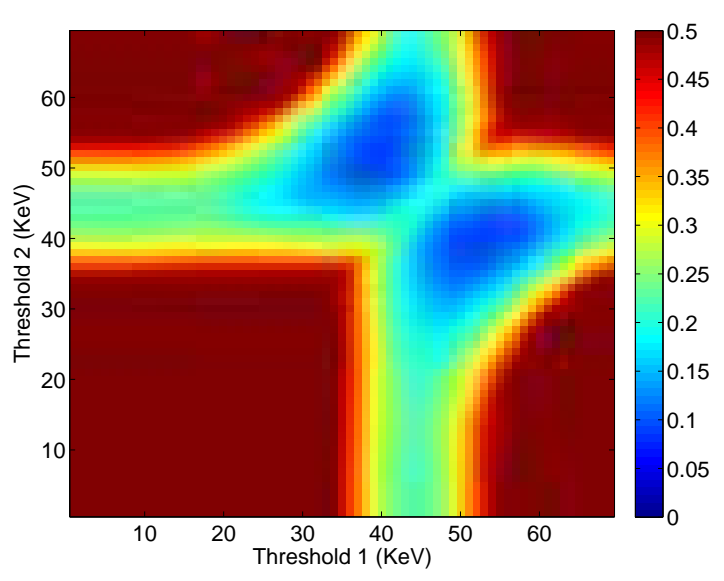

(b)

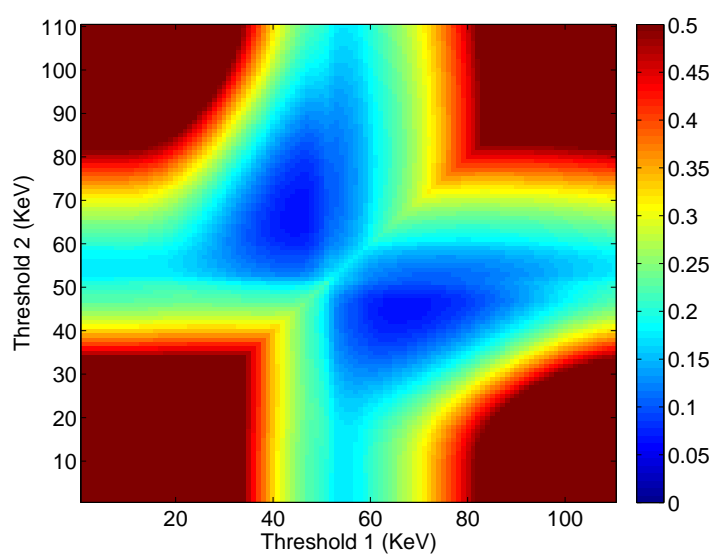

Figure 2: Simulated $L_{1}$ distances of spectrum binning with 2 (left) and 3 (right) energy bins using the thresholdbased method for the 80 and $120 \mathrm{kVp}$ spectra.

(f) Proposed physics-based polychromatic reconstruction using generalized spectrum binning (PSR-GSB2 and PSR-GSB3).

A separable quadratic surrogate and ordered $\operatorname{subset}^{2}$ (32 subsets) are used to solve the optimization problem for all statistical iterative reconstruction methods. The number of iterations for all iterative reconstruction is 100. The Huber penalty function of 4 neighboring pixels was used. The transition parameter of the Huber function between the quadratic and linear regions is $5 \mathrm{HU}$.

\section{RESULTS}

Figure 2 shows the $L_{1}$ distances of the threshold-based spectrum binning. The values at the boundaries are equivalent to using only one bin. Using more than one energy leads to much smaller beam hardening errors than the monochromatic assumption (a single bin). Additionally, more energy bins can help to reduce the difference between true polychromatic and approximated signals.

Figure 3 shows the attenuation errors for different material thicknesses using the proposed spectrum binning methods. With constrains on the sum of bin sizes, the error of the detector expected signal is zero when there is no soft tissue and bone. Error in the approximate line integral increases as the material thickness increases. Using three energy bins has smaller errors than using two bins. The GSB approach has much smaller error than the threshold-based SB approach. Table 1 shows the resulting values of $B_{s}, \Phi_{s}, \Theta_{s}$ of the threshold-based spectrum binning (SB) and the generalized spectrum binning (GSB). Again, the distances of generalized spectrum binning 


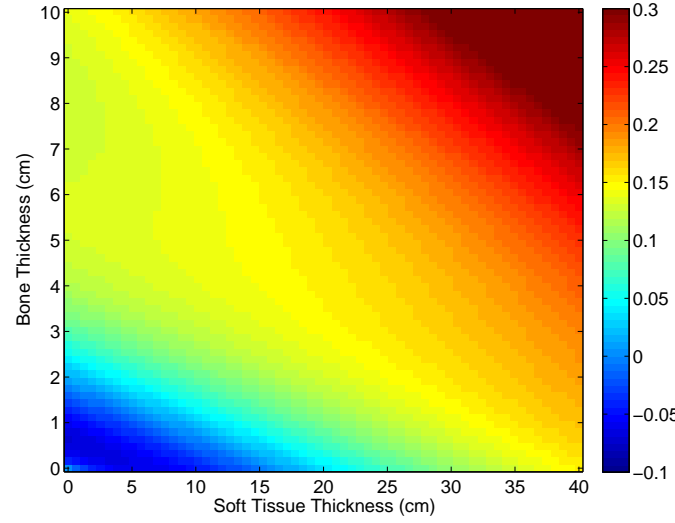

(a) SB2

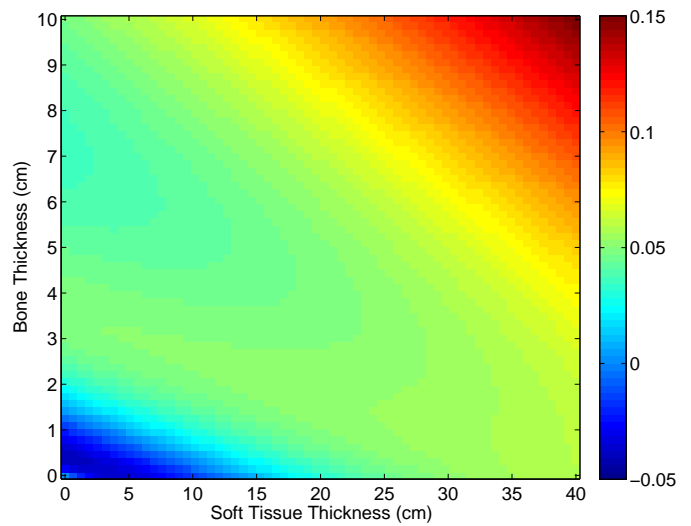

(c) SB3

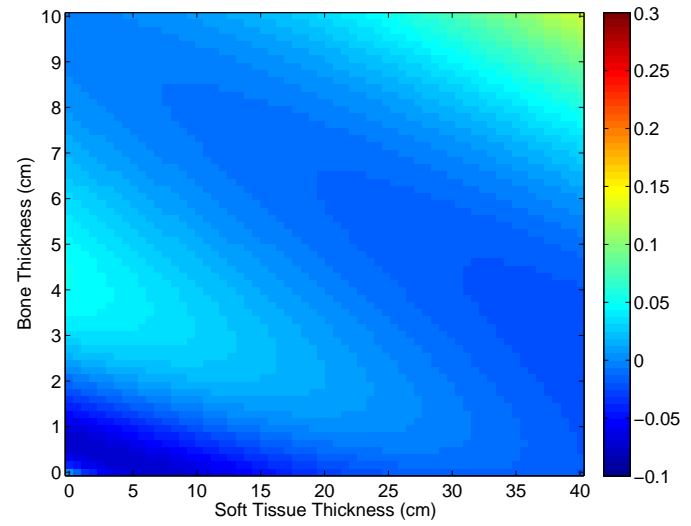

(b) GSB2

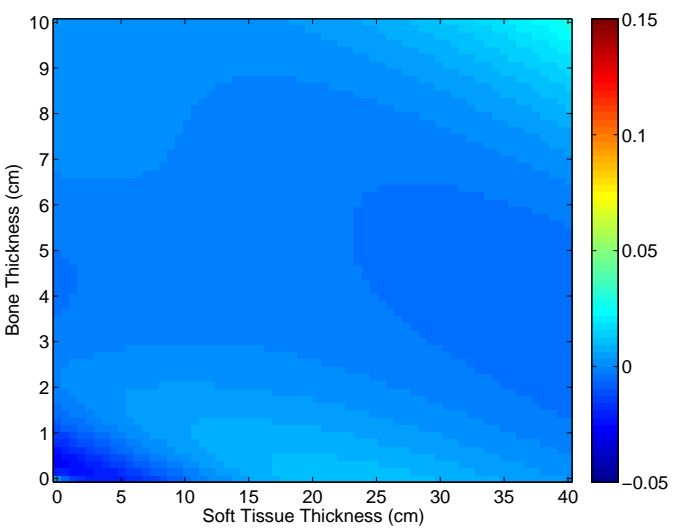

(d) GSB3

Figure 3: Attenuation errors at different material thickness using proposed spectrum binning methods for the 120 spectrum. Note change in scale between SB2, GSB2 and SB3, GSB3 plots.

are much smaller than the threshold-based spectrum binning. Three bins have smaller distances than 2 bins for both the binning methods.

Table 1: Spectrum binning results for 0-40 cm soft tissue and 0-10 cm cortical bone.

\begin{tabular}{|c|l|l|l|l|l|}
\hline Spectrum & Method & $B_{s}$ (normalized) & $\Phi_{s}$ & $\Theta_{s}$ & $L_{1}$ distance \\
\hline $80 \mathrm{kVp}$ & SB2 & $0.74,0.26$ & $1.5330,0.3659$ & $1.0182,0.9503$ & 0.1926 \\
\hline $80 \mathrm{kVp}$ & GSB2 & $0.68,0.32$ & $1.6842,0.3742$ & $1.0251,0.9444$ & 0.0192 \\
\hline $80 \mathrm{kVp}$ & SB3 & $0.62,0.23,0.15$ & $1.8060,0.5542,0.3052$ & $1.0248,0.9720,0.9402$ & 0.0528 \\
\hline $80 \mathrm{kVp}$ & GSB3 & $0.45,0.40,0.25$ & $2.4077,0.6998,0.2810$ & $1.0368,0.9862,0.9338$ & 0.0026 \\
\hline $120 \mathrm{kVp}$ & SB2 & $0.70,0.30$ & $1.8655,0.3414$ & $1.0408,0.9381$ & 0.1481 \\
\hline $120 \mathrm{kVp}$ & GSB2 & $0.62,0.38$ & $2.1332,0.3723$ & $1.0410,0.9303$ & 0.0153 \\
\hline $120 \mathrm{kVp}$ & SB3 & $0.51,0.34,0.15$ & $2.6732,0.6676,0.2302$ & $1.0468,0.9780,0.9126$ & 0.0374 \\
\hline $120 \mathrm{kVp}$ & GSB3 & $0.38,0.46,0.16$ & $3.636,0.8096,0.2160$ & $1.0603,0.9919,0.9049$ & 0.0027 \\
\hline
\end{tabular}

Figures 4 and 5 show reconstructed images using the reconstruction methods listed. The beam hardening artifacts are suppressed when using the polychromatic statistical reconstruction methods. Similar to the results in Figure 3, using more energy bins and the GSB approach suppresses more beam hardening artifacts. The generalized spectrum binning with three bins can effectively reduce the beam hardening artifacts in the images. Table 2 summarizes the soft tissue standard deviation of the reconstructed images in Figures 4 and 5 . The noise 


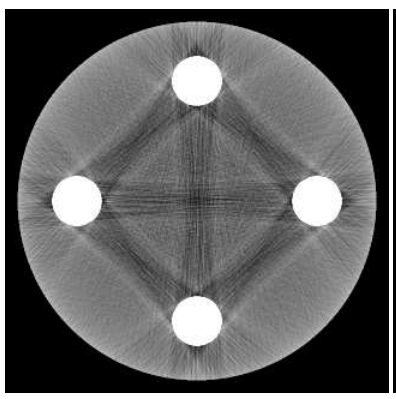

(a) FBP-WC

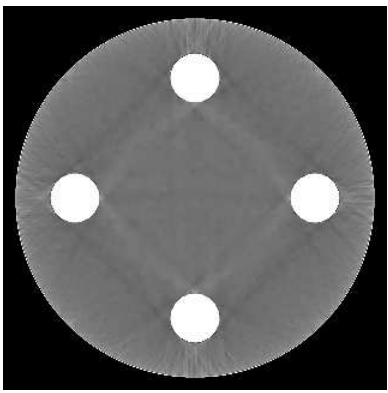

(e) PSR-SB2

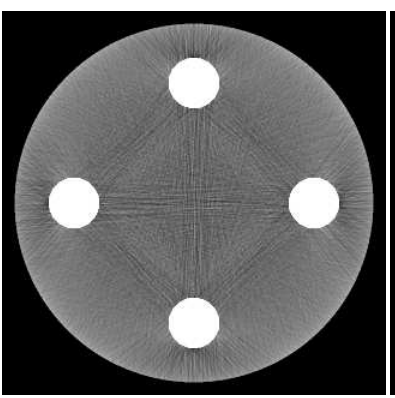

(b) FBP-JS

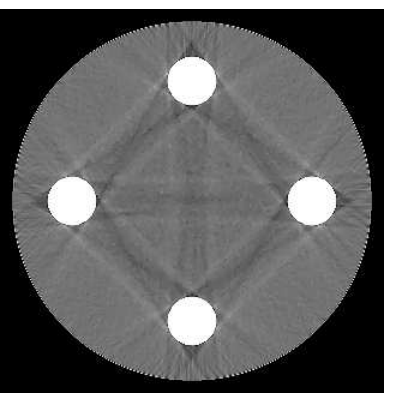

(c) PWLS

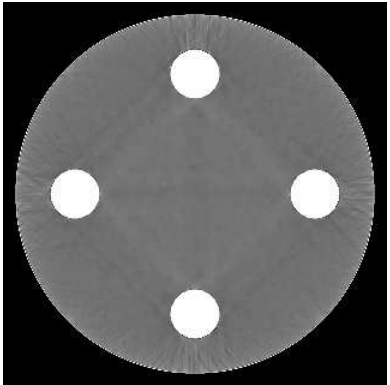

(f) PSR-SB3

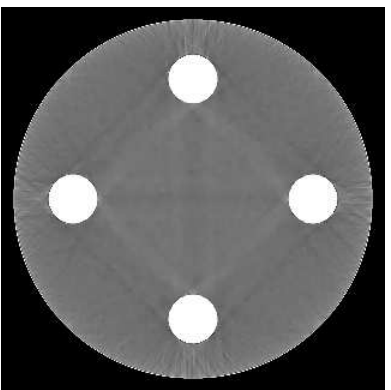

(g) PSR-GSB2

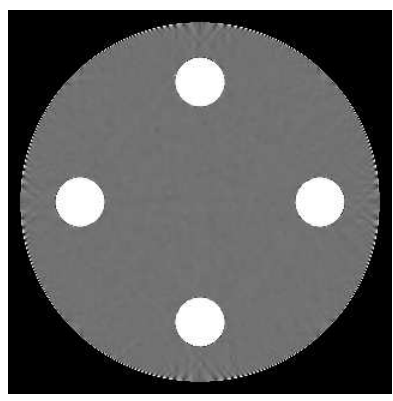

(d) PSR-full

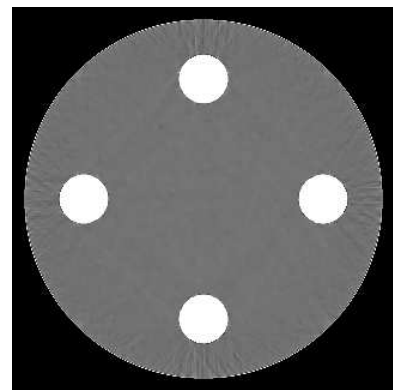

(h) PSR-GSB3

Figure 4: Image reconstruction results using $80 \mathrm{kVp}$ spectrum. The display window is [-200 400] HU.

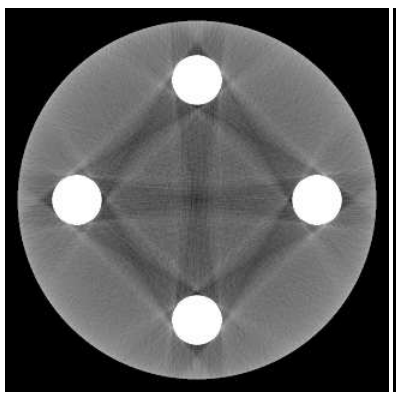

(a) FBP-WC

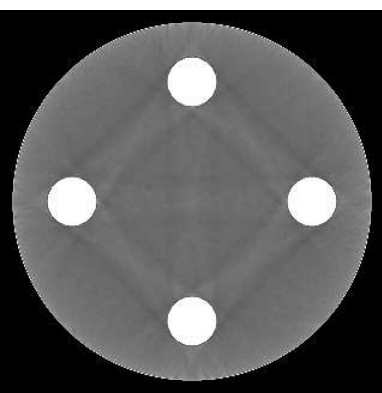

(e) PSR-SB2

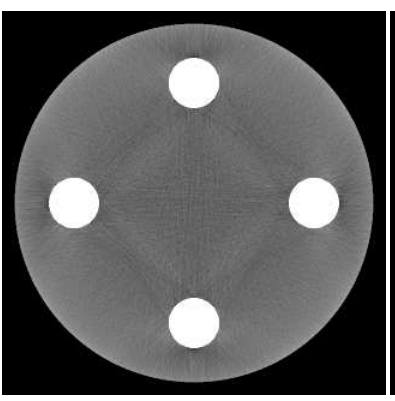

(b) FBP-JS

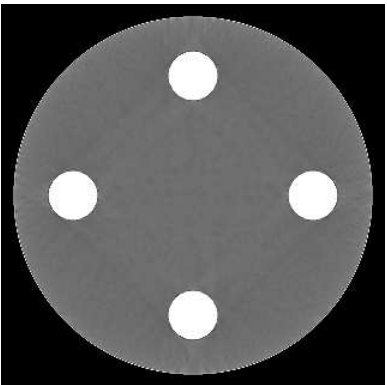

(f) PSR-SB3

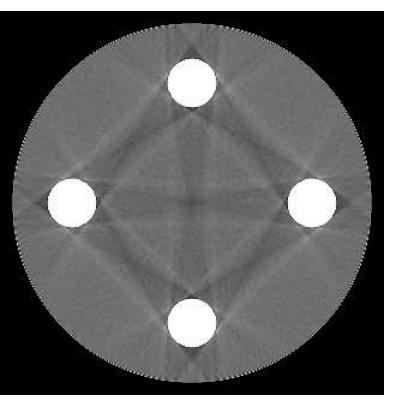

(c) PWLS

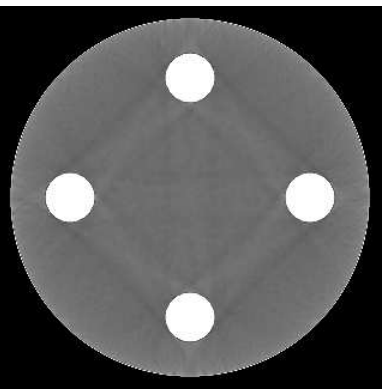

(g) PSR-GSB2

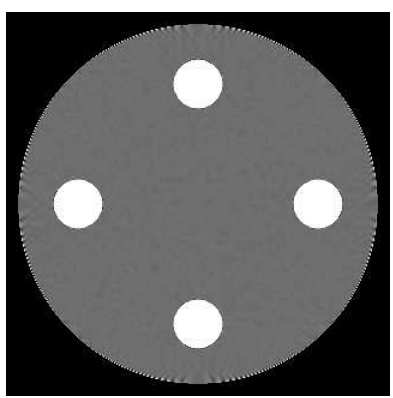

(d) PSR-full

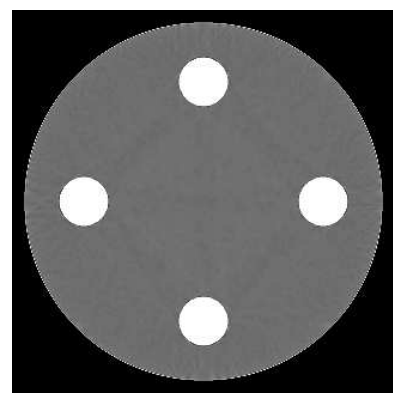

(h) PSR-GSB3

Figure 5: Image reconstruction results using $120 \mathrm{kVp}$ spectrum. The display window is [-200 400] HU.

in the middle of the images is high, because the x-rays are highly attenuated by the bone columns. Statistical reconstruction methods can reduce the noise level, with the result that the reconstructed images look smoother than those from the FBP reconstruction. Figure 6 shows the line profiles of the central row. The line profile of the PSR-GSB3 is almost the same as PSR-full. 


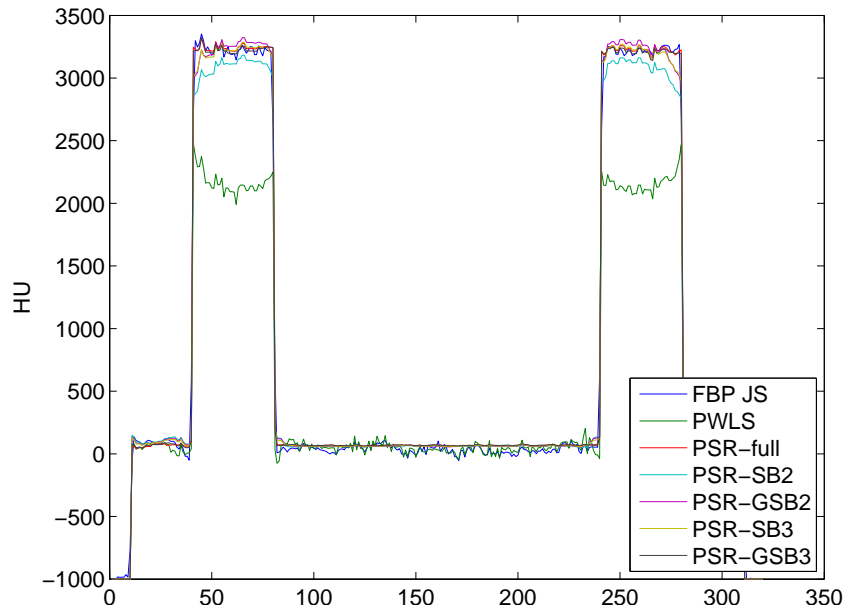

(a) $80 \mathrm{kVp}$

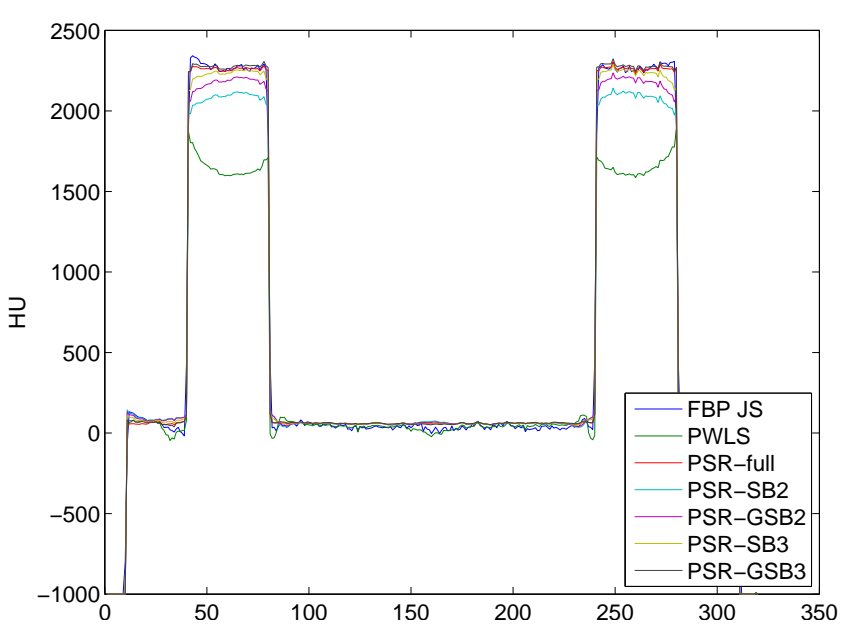

(b) $120 \mathrm{kVp}$

Figure 6: Central row profiles in $\mathrm{HU}$ of reconstructed images.

Table 2: Quantitative evaluations of reconstructions. Soft tissue standard deviation in HU.

\begin{tabular}{|l|c|c|}
\hline Algorithm & Soft tissue STD $(80 \mathrm{kVp})$ & Soft tissue STD $(120 \mathrm{kVp})$ \\
\hline FPB-WC & 48.4 & 37.7 \\
\hline FPB-JS & 29.5 & 20.8 \\
\hline PWLS & 18.4 & 18.0 \\
\hline PSR-full & 4.5 & 3.8 \\
\hline PSR-SB2 & 11.5 & 12.0 \\
\hline PSR-GSB2 & 9.5 & 9.4 \\
\hline PSR-SB3 & 8.3 & 5.7 \\
\hline PSR-GSB3 & 5.8 & 4.4 \\
\hline
\end{tabular}

\section{CONCLUSIONS AND DISCUSSION}

We have proposed a modified optimization problem for polychromatic statistical reconstruction algorithms, which is relatively easier to solve while being applicable to multi-material datasets. In addition, a spectrum binning method is developed to reduce the prior knowledge required from the full spectrum to only the ratios between several energy bins. Since only the ratios of energy bins sizes matter, there are no additional inputs and computation loads. From simulation studies, it is shown that three energy bins using the generalized spectrum binning algorithm have an average absolute error of the logarithmic signal less than 0.003 . The proposed modified optimization problem and spectrum binning method can effectively suppress beam hardening artifacts while providing low noise in the reconstructed images.

By adjusting the bin sizes for different spectra, the algorithm can be applied with bowtie filters, which has not been considered by most existing statistical methods. Moreover, the spectrum binning approach can also be based on the scan object. A prior reconstruction may be used to determine weights of the material thickness in computing the distances. The weighted distance based on the scan object may provide more efficient spectrum binning results than using even weights over certain ranges.

\section{REFERENCES}

[1] Joseph, P. M. and Spital, R. D., "A method for correcting bone induced artifacts in computed tomography scanners," Journal of computer assisted tomography 2, 100-8 (Jan. 1978).

[2] Elbakri, I. A. and Fessler, J. A., "Statistical image reconstruction for polyenergetic X-ray computed tomography," IEEE Trans Med Imaging 21, 89-99 (Feb. 2002). 
[3] De Man, B., Nuyts, J., Dupont, P., Marchal, G., and Suetens, P., "An iterative maximum-likelihood polychromatic algorithm for CT.," IEEE transactions on medical imaging 20, 999-1008 (Oct. 2001).

[4] Menvielle, N., Goussard, Y., Orban, D., and Soulez, G., "Reduction of Beam-Hardening Artifacts in X-Ray CT," in [Annual International Conference of the IEEE Engineering in Medicine and Biology Society.], 2, 1865-8 (Jan. 2005).

[5] Van Gompel, G., Van Slambrouck, K., Defrise, M., Batenburg, K. J., de Mey, J., Sijbers, J., and Nuyts, J., "Iterative correction of beam hardening artifacts in CT," Medical physics 38 Suppl 1, S36 (July 2011).

[6] Yang, Q., Elter, M., Schasiepen, I., Maass, N., and Hornegger, J., "Fast iterative beam hardening correction based on frequency splitting in computed tomography," in [SPIE Medical Imaging], Nishikawa, R. M. and Whiting, B. R., eds., 8668, 86682J (Mar. 2013).

[7] Yan, C. H., Whalen, R. T., Beaupré, G. S., Yen, S. Y., and Napel, S., "Reconstruction algorithm for polychromatic CT imaging: application to beam hardening correction.," IEEE transactions on medical imaging 19, 1-11 (Jan. 2000).

[8] Elbakri, I. A. and Fessler, J. A., "Segmentation-free statistical image reconstruction for polyenergetic x-ray computed tomography with experimental validation," Physics in medicine and biology 48, 2453-77 (Aug. 2003).

[9] Hubbell, J. H. and Seltzer, S. M., "Tables of X-Ray Mass Attenuation Coefficients and Mass EnergyAbsorption Coefficients from $1 \mathrm{keV}$ to $20 \mathrm{MeV}$ for Elements $\mathrm{Z}=1$ to 92 and 48 Additional Substances of Dosimetric Interest," tech. rep., National Institute of Standards and Technology (Available: http://physics.nist.gov/xaamdi), Gaithersburg, MD. (2004). 